died, and no differences were noted in the survivaltimes or symptoms of the animals in the two groups.

With these doses of histamine, also, approximately the LD 50 under our conditions, we could thus not repeat Traina's results.

Blood-Pressure. (Cat, anæsthetized with intravenous chloralose, $80 \mathrm{mgm}$. $/ \mathrm{kgm}$.). Three intravenous doses of histamine were given at 2 -min intervals. This procedure was repeated at intervals after the intravenous administration of vitamin $B_{12}$. The fall of arterial blood pressure following each histamine injection was recorded, and the mean fall (in $\mathrm{mm}$. mercury) was estimated for each group of three injections. The results are presented in Table 2.

From these observations it may be concluded that injection of $50-500 \mu \mathrm{gm} . \mathrm{B}_{12}$ ('Cytamen', Glaxo) per $\mathrm{kgm}$. has no effect on the depressor effect of histamine in the cat under chloralose anæsthesia.

$$
\begin{gathered}
\text { H. M. SharPe } \\
\text { E. A. WoolletT } \\
\text { W. F. J. CuthBertson } \\
\text { Research and Development Division, } \\
\text { Glaxo Laboratories, Limited, } \\
\text { Greenford, } \\
\text { Middlesex. }
\end{gathered}
$$

1 Traina, V., Nature, 165, 439 (1950).

\section{Occurrence of Eburicoic Acid}

IN the course of extensive studies on the metabolic products formed by the higher fungi, mainly of the class Basidiomycetes, grown on synthetic media, we examined a sample of decayed Eucalyptus regnans wood discovered at the Australian Newsprint Mills, Boyer, Tasmania, in 1944. From this mass of rotted wood encompassed with mycelial hyphr, the acetyl derivative of a monobasic acid has been isolated. On hydrolysis this acetate (m.p. $256-257^{\circ} ;[\alpha]_{D}^{20}+35 \cdot 8^{\circ}$ $(c, 3 \cdot 1$ in pyridine) ; found: $\mathrm{C}, 75 \cdot 9 ; \mathrm{H}, 10 \cdot 1$ per cent ; molec. wt. (Rast), $455 ; \mathrm{C}_{29} \mathrm{H}_{46} \mathrm{O}_{4}$ requires $\mathrm{C}$, $76.0 ; \mathrm{H}, 10 \cdot 1$ per cent; molec. wt. 458) gave rise to the parent acid (m.p. $293^{\circ},[\alpha]_{D}^{17}+35 \cdot 6^{\circ}(c, 1 \cdot 4$ in pyridine); found: $\mathrm{C}, 77.9 ; \mathrm{H}, 10.5 ; \mathrm{C}_{27} \mathrm{H}_{44} \mathrm{O}_{3}$ requires $\mathrm{C}, 77.9 ; \mathrm{H}, 10.6$ per cent), which appears to be a $\mathrm{C}_{27^{-}}$and not the more usual $\mathrm{C}_{30^{-}}$type of the triterpene group, examples of which are known to occur in naturally grown Polyporos betulinus Fr. ${ }^{1}$.

From the decayed wood the organism responsible for this 'white rot' was isolated and identified as Polyporus anthracophilus Cooke and, when grown on a standard synthetical medium, containing glucose and glycine, was found to produce the parent acid (isolated from the mycelium) and not the acetate. From the literature it appeared that the acid from $P$. anthracophilus Cooke was in all probability identical with eburicoic acid, isolated along with agaracinic acid from the naturally grown fungus Fomes officinalis Fr., by Kariyone and Kurono ${ }^{2}$, who give the melting point as $283^{\circ}$ for their compound, which they named eburicoic acid and considered to have formula $\mathrm{C}_{30} \mathrm{H}_{48} \mathrm{O}_{3}$; according to these authors, the acetyl derivative had melting point $240^{\circ},[\alpha]_{D}^{17}+80^{\circ}$, the methyl ester, melting point $141^{\circ},[\alpha]_{D}^{21}+37^{\circ}$, and the acetate of the methyl ester, melting point $150^{\circ}$, $[\alpha]_{D}^{22}+56.9^{\circ}$. When grown on the synthetical medium, this mould has been found to produce the acid (m.p. $293^{\circ}$ ) ; but in this instance the crude compound is contaminated with a product of similar composition, having an ultra-violet absorption spectrum, maximum at $243 \mathrm{~m} \mu, E^{1} \underset{1 \mathrm{~cm} \text {. }}{\text { per }} 37 \cdot 8$. From this mixture the acid (m.p. $293^{\circ}$ ) has been separated by chromatography on a column of aluminium oxide neutralized by acetic acid - methanol mixture. Although Kariyone and Kurono employed a hydrolysis process in the isolation of their eburicoic acid, it is not clear whether the naturally grown mould gives the free acid or the acetate. In spite of this difference in the melting points of Kariyone and Kurono's eburicoic acid and the acid produced by the moulds grown under artificial conditions and of some of their respective derivatives, we believe them to be identical.

In addition to the foregoing moulds, eburicoic acid has also been isolated from the mycelium of Polyporus eucalyptorum Fr., P. sulfureus (Bull) Fr., and Lentinus dactyloides Cleland. The following derivatives have been prepared in the course of the identification of eburicoic acid from various sources: methyl eburicoate (m.p. 140-141 ${ }^{\circ}$; found : C, 78.1 ; H, 10.8; $\mathrm{C}_{28} \mathrm{H}_{48} \mathrm{O}_{3}$ requires $\mathrm{C}, 78.2 ; \mathrm{H}, 10 \cdot 7$ per cent), methyl

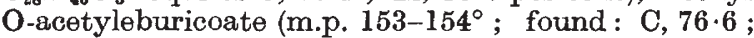
$\mathrm{H}, 10.4 ; \mathrm{OMe}, 5.3 ; \mathrm{C}_{28} \mathrm{H}_{45} \mathrm{O}_{2}(\mathrm{OM} \theta)$ requires $\mathrm{C}$, $\mathbf{7 6} \cdot 3 ; \mathrm{H}, 10 \cdot 2 ; \mathrm{OMe}, 6 \cdot 6$ per cent) and $p$-nitrobenzyl eburicoate (m.p. $150 \cdot 5^{\circ}$; found: C, $74 \cdot 2 ; \mathrm{H}, 9 \cdot 1$; $\mathrm{N}, 2.4 ; \mathrm{C}_{34} \mathrm{H}_{48} \mathrm{O}_{5} \mathrm{~N}$ requires $\mathrm{C}, 74.1 ; \mathrm{H}, 9.0 ; \mathrm{N}$, $2 \cdot 5$ per cent). Experiments on the constitution of eburicoic acid are in progress.

\section{R. M. Gascoigne \\ J. S. E. HOLKER \\ B. J. RALPH}

ALEXANDER RoBertson

Department of Organic Chemistry,

University of Liverpool. July 28 .

${ }^{1}$ Cross, Fliot, Heilbron and Jones, J. Chem. Soc., 632 (1940).

2 Kariyone and Kurono, J. Pharm. Soc. Japan, 60, 110, 318 (1940).

\section{The de Haas-van Alphen Effect}

Experiments by one of us ${ }^{1}$ on the de Haas-van Alphen effect in gallium, tin and graphite (periodic field-dependence of magnetic susceptibility of metal single crystals) have now been extended to higher fields (up to 15,800 gauss), to lower temperatures (down to $1 \cdot 1^{\circ} \mathrm{K}$.), and to other metals; at the same time the theory of the effect has been reconsidered. The present note gives a brief account of the new results.

The effect has been shown to occur along the hexagonal axis of cadmium and perpendicular to the tetragonal axis of indium ; for both metals the period of oscillation is very small (at 15,300 gauss about 43 gauss for cadmium and $\mathbf{4 8}$ gauss for indium). The amplitude is scarcely measurable except at the highest fields and lowest temperatures, so it is not surprising that the effect was not observed in the earlier experiments. Similar results have been found quite recently by Verkin, Lazarev and Rudenko ${ }^{2}$, who have also observed the effect in magnesium and beryllium. More thorough experiments on a pure gallium crystal have shown that the effect is more marked and its character more complicated than the previous observations on a less pure crystal had suggested; the characteristics of the effect are different for each of the three principal axes. One interesting feature (which occurs also for tin and graphite) is the existence of beats in the oscillations, that is, periodic modulation of the envelope of the oscillations. The beat period varies in a complicated manner with field direction, and the depth of modula- 